\title{
Polymer optical fibers doped with organic materials as luminescent solar concentrator
}

\author{
Itxaso Parola ${ }^{\text {a }}$, María Asunción Illarramendi ${ }^{a}$, Joseba Zubia ${ }^{\mathrm{a}}$, Eneko Arrospide ${ }^{\mathrm{a}}$, Gaizka Durana ${ }^{\mathrm{a}}$, \\ Nekane Guarrotxena ${ }^{b}$, Olga García ${ }^{b}$, Robert Evert ${ }^{c}$, Daniel Zaremba $^{c}$, Hans-Hermann Johannes ${ }^{c}$, \\ Federico Recart ${ }^{\mathrm{d}}$ \\ ${ }^{a}$ University of the Basque Country (UPV/EHU), Faculty of Engineering of Bilbao (EIB), Plaza \\ Ingeniero Torres Quevedo 1, E-48013 Bilbao, Spain \\ ${ }^{\mathrm{b}}$ Instituto de Ciencia y Tecnología de Polímeros (ICTP), Consejo Superior de Investigaciones \\ Científicas (CSIC), Juan de la Cierva 3, Madrid 28006, Spain \\ ${ }^{c}$ Technische Universität Braunschweig, Institut für Hochfrequenztechnik, Labor für Elektrooptik, \\ Bienroder Weg 94, 38106 Braunschweig, Germany \\ ${ }^{\mathrm{d} I n s t i t u t o ~ d e ~ T e c n o l o g i ́ a ~ M i c r o e l e c t r o ́ n i c a, ~ P a r q u e ~ T e c n o l o g i c o ~ d e ~ B i z k a i a, ~} 48170$ Zamudio, Spain
}

\begin{abstract}
This paper provides an analysis of the emission properties of different polymer optical fibers doped with organic materials with the aim of investigate their performance as luminescent solar concentrators. We present a study of the light propagation along the fibers, together with the experimental measurements of two different efficiencies: side illumination coupling efficiency and fluorescent fiber solar concentrator efficiency. The results obtained for all the fibers are compared and discussed.
\end{abstract}

Keywords: Polymer optical fiber, Organic materials, Fluorescent and luminescent materials, Fluorescent fiber solar concentrator, Luminescent solar concentrator.

\section{INTRODUCTION}

In recent years, photovoltaic energy has attracted a great deal of attention due to its potential as abundant, inexhaustible, climate friendly and ubiquitous energy source. The goal of converting sunlight power directly into electricity using photovoltaic cells has occupied researchers for the last fifty years, with the silicon-based solar cell dominating the field. However, collecting sunlight in an efficient and especially economical way remains one of the main goals of photovoltaic technology. A possible approach to reduce the cost of the harvesting system is by concentrating the sunlight onto a small area of the expensive solar cells. First conventional solar concentrators based on mirrors and lenses required precise tracking mechanism and they were not suitable for cloudy weather and diffuse light conditions. Luminescent solar concentrators (LSC) were then designed as a diffuse light collector and as a static solution. It was in the late 1970s when the concept of LSC was proposed by Weber and Lambe [1].

LSC consist of a layer of transparent medium containing luminescent species which absorb part of the solar radiation and re-emit it in higher wavelengths, also known as down-shifting process. A fraction of the photons emitted by the fluorescent material are trapped and transported by total internal reflection to the edge of the layer where the solar cells are attached. Due to the location of the solar cells on the edges of the luminescent layer, it comprises a much smaller area, and the fact that the cost of transparent polymers such as poly-methylmethacrylate (PMMA) is much lower than that of the solar cell, the overall cost of the photovoltaic energy production is considerably decreased. However, the main drawback of the planar LSC is the limitation on the coupling between the layer and the solar cell and the difficult wiring method for light transportation. A new concept of LSC appears to be a competent solution targeting on the aforementioned limitations: fluorescent fiber solar concentrator (FFSC). The cylindrical geometry of the fibers, allows an easy coupling between the fluorescent fiber and the solar cells, reducing the coupling losses, and they can also be easily attached to transparent optical fibers for light transportation, overcoming the biggest disadvantage of the planar LSC. Some theoretical studies have been carried out in the last years involving comparisons between cylindrical and planar LSCs [2-4]. In the research developed by K.R. McIntosh et al. it was found that the optical concentration of a cylindrical

Organic Photonic Materials and Devices XIX, edited by Christopher E. Tabor, François Kajzar,

Toshikuni Kaino, Yasuhiro Koike, Proc. of SPIE Vol. 10101, 101010Z · C 2017 SPIE

CCC code: $0277-786 X / 17 / \$ 18 \cdot$ doi: $10.1117 / 12.2252956$ 
LSC is 1-1.9 times greater than an equivalent planar LSC, and the cylindrical LSC requires a smaller area of solar cells to produce the same amount of electricity as an equivalent square LSC [3]. It has also been found that for the proper functioning of a FFSC, the luminescent molecules must absorb and re-emit light very close to the surface. For this reason, clad-only doped fibers have started to be investigated [5,6]. Recently a study on different fiber structures has been published targeting on the increase on the efficiency of the FFSC As for the luminescent materials employed as dopant, plenty of different species have been studied for the planar LSC, from organic fluorescent dyes to quantum dots and rare earth complexes [7-12]. In order for LSCs to achieve light concentration efficiencies greater than $10 \%$ when used with silicon solar cells, there are four main criteria that should be met: absorption of all the $\lambda<950 \mathrm{~nm}$ and emission peaks around $1000 \mathrm{~nm}$, minimum reabsorption losses, near unity fluorescent quantum yields and long term stability [13]. Some organic materials such as the derivatives of perylene dye exhibit near unity quantum yields and have been demonstrated to last stable for many years in PMMA hosts [14]. However, little studies on different dopants have been carried out yet for FFSCs.

The aim of this work is to carry out a total optical characterization of several polymer optical fibers (POF) doped with organic dyes and conjugated polymers, for future FFSC applications. The study includes a spectroscopic characterization of the light propagation distance along the fiber, and a calculation of the side illumination absorption efficiency and the fluorescent fiber solar concentration efficiency. The results obtained have been thoroughly compared, and interesting conclusions have been obtained for upcoming works on the field of FFSC.

\section{EXPERIMENTAL}

The fibers analyzed are summarized in Table 1. The first sample, a polymer optical fiber (POF) doped with Lumogen Orange (LO), was manufactured in the drawing tower of the Applied Photonics Group Bilbao (APG Bilbao) with the collaboration of the Spanish National Research Council (CSIC). The preform was produced by an extrusion process, where the fully polymerized poly-(methylmethacrylate) (PMMA) and the dopant are fed into a reaction chamber. This material is pushed through a small nozzle and finally a preform of $11 \mathrm{~mm}$ is obtained. After that, the preform was annealed during 2 weeks in a climate chamber before drawing it to a $1 \mathrm{~mm}$ fiber using the POF-drawing tower at a maximum furnace temperature of $185{ }^{\circ} \mathrm{C}$. Lumogen Red doped samples (LR01-04) were manufactured by our collaborators from Technical University Braunschweig (TU Braunschweig). The preforms were polymerized in bulk, filling borosilicate tubes of $10 \mathrm{~mm}$ with a nitrogen saturated solution of methyl-methacrylate, Lumogen F Red 300 in different concentrations, lauroyl peroxide $(0.03 \mathrm{~mol} \%)$ and 1-butyl-mercaptan $(0.2 \mathrm{~mol} \%)$. These tubes were correctly sealed and transferred to a heating cabinet, where they were heated slowly to $100{ }^{\circ} \mathrm{C}$ over five days and were maintained at a constant temperature of $100{ }^{\circ} \mathrm{C}$ for another two days. Then, they heated up to $230^{\circ} \mathrm{C}$ in the heater unit of the drawing tower and drawn to fiber by applying a constant force. In the case of the cladded fiber, a film of $20 \mu \mathrm{m}$ of commercial Fospia PC-404F AP material was applied through a nozzle and cured by UV-irradiation resulting in a numerical aperture of 0.5 . The last three fibers are doped with three different conjugated polymers, namely poly(9,9-dioctylfluorene)-altbenzothiadiazole (F8BT), poly(9,9-dioctylfluorene-alt-bithiophene) (F8T2), and poly[2,7-(9,9bis(2'ethylhexyl)fluorene)alt-2,5-terthiophene] (PF3T). They were produced by the POF manufacturing company Luceat S.p.A (Italy) using an adapted preform-drawing technique, which further details are described in [15].

Table 1. Summary of the characteristics of the fibers analyzed.

\begin{tabular}{|c|c|c|c|c|c|c|}
\hline Code & Dopant & Concentration & Type & $\emptyset$ & Matrix & Manufacturing \\
\hline LO & Lumogen F Orange 240 & $0.003 \mathrm{~mol} \%$ & SI/OC & $1 \mathrm{~mm}$ & PMMA & APG Bilbao/CSIC \\
\hline LR01 & Lumogen F Red 300 & $0.0001 \mathrm{~mol} \%$ & SI/WC & $1 \mathrm{~mm}$ & PMMA & TU Braunschweig \\
\hline LR02 & Lumogen F Red 300 & $0.0005 \mathrm{~mol} \%$ & SI/WC & $1 \mathrm{~mm}$ & PMMA & TU Braunschweig \\
\hline LR03 & Lumogen F Red 300 & $0.03 \mathrm{~mol} \%$ & SI/WC & $1 \mathrm{~mm}$ & PMMA & TU Braunschweig \\
\hline LR04 & Lumogen F Red 300 & $0.03 \mathrm{~mol} \%$ & SI/OC & $1 \mathrm{~mm}$ & PMMA & TU Braunschweig \\
\hline
\end{tabular}




\begin{tabular}{|c|c|c|c|c|c|c|}
\hline F8BT & $\begin{array}{c}\text { poly(9,9-dioctylfluorene)-alt- } \\
\text { benzothiadiazole }\end{array}$ & $0.003 \mathrm{~mol} \%$ & SI/OC & $1 \mathrm{~mm}$ & PMMA & Luceat S.p.A \\
\hline F8T2 & $\begin{array}{c}\text { poly(9,9-dioctylfluorene-alt- } \\
\text { bithiophene) }\end{array}$ & $0.003 \mathrm{~mol} \%$ & SI/OC & $1 \mathrm{~mm}$ & PMMA & Luceat S.p.A \\
\hline PF3T & $\begin{array}{c}\text { poly[2,7-(9,9-bis(2'- } \\
\text { ethylhexyl)fluorene)-alt-2,5- } \\
\text { terthiophene] }\end{array}$ & $0.003 \mathrm{~mol} \%$ & SI/OC & $1 \mathrm{~mm}$ & PMMA & Luceat S.p.A \\
\hline
\end{tabular}

*SI: step-index; OC: only-core; WC: with cladding

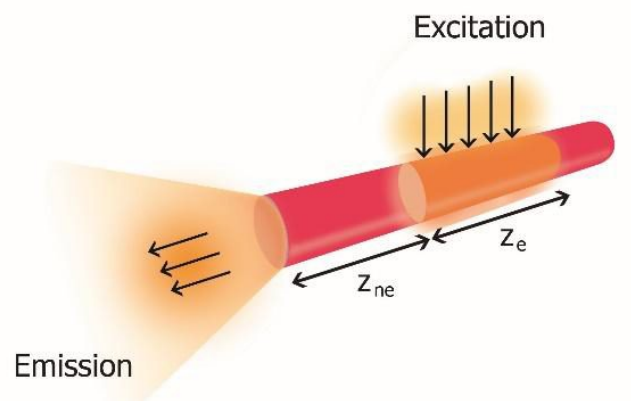

Figure 1. Schematic of the transversal excitation using the side illumination technique. $\mathrm{z}_{\mathrm{e}}$ : transversally excited length of the fiber; $\mathrm{z}_{\mathrm{ne}}$ : non-excited length of the fiber up to the detector.

The samples have been transversally pumped using the Side Illumination Technique (SIT) (Figure 1.). This method is a non-destructive technique for measuring the optical losses and it also allows a characterization of the effects of the propagation distance along the fiber. The main analysis of this work has been carried out by using transversal excitation in a spot of $1.2 \mathrm{~mm}$ of diameter employing a tunable ultrafast femtosecond laser (Spectra Physics Mai Tai HP) with a wavelength range between 690 and $1040 \mathrm{~nm}$ and $80 \mathrm{MHz}$ of repetition rate. This laser emits Gaussian pulses with a temporal width of around $100 \mathrm{fs}$. The optical pumping at the desired wavelengths in the visible region was obtained using the output signal of a frequency-doubler (Radiantis Inspire Blue). The pulse power was controlled by adjusting a beam splitter, positioned between the laser and the frequency-doubler. The acquisition of the emission spectra has been performed using two different detectors. For the time resolved spectroscopy, an ultrafast Streak Camera detector with a resolution of $10 \mathrm{ps}$ was used (Hamamatsu C5680-21). This detector operates in a spectral range from 200 to $800 \mathrm{~nm}$. The device comprises a delay unit for the proper synchronization of the acquisition. For the steady-state measurements, a fiber-optic spectrometer (Ocean Optics USB4000, optical resolution of $1.5 \mathrm{~nm}$ of full width at half maximum) mounted on a linear stage driven by a motor controlled was used. For the side illumination absorption efficiency calculations, a silicon detector with a calibration module (Newport 818-SL/DB Silicon Photodetector) was employed. For the solar concentration efficiency measurements a halogen lamp (OSRAM, Haloline Eco) was used. The fiber was excited from the top for different fiber lengths. The detection was made employing a AvaSpec-2048 fiber optic spectrometer and the above-mentioned silicon detector taking into account the spectral responsivity curve of the latter.

\section{RESULTS AND DISCUSSION}

\subsection{Spatial and temporal characterization}

In this subsection we present the temporal and spatial features of the emission of the fibers. The samples were transversally excited at different wavelengths with low pump irradiance. An example of the absorption and emission spectra of two different fibers is shown in Figure 2(a), where the overlap between both curves of the organic dyes can be observed. The values of the stokes shift of each fiber are gathered in Table 2. For the temporal characterization, the samples were pumped at two different excitation wavelengths, $\lambda_{\mathrm{ex}}$, namely $520 \mathrm{~nm}$ and $457 \mathrm{~nm}$. As an example, the 
fluorescence decay of the LO fiber is represented in Figure 2(b), for an integration time of $20 \mathrm{~ns}$. As can be seen, the electrons are relaxed to the ground state in a process well reproduced with an exponential decay equation. This exponential trend has been observed for all the samples. The life-time values are obtained by the fittings of the experimental data, and they are also collected in Table 2. From this analysis, no variations in the temporal life-times have been found for different propagation distances in none of the fibers. It has also been demonstrated that these values remain invariable as well when the excitation wavelength is changed and when different emission wavelengths are analyzed. Therefore, we can reach to the conclusion that the temporal emission of the fibers studied in this work is not wavelength dependent, nor propagation distance dependent, at least for the lengths analyzed in this experiment.
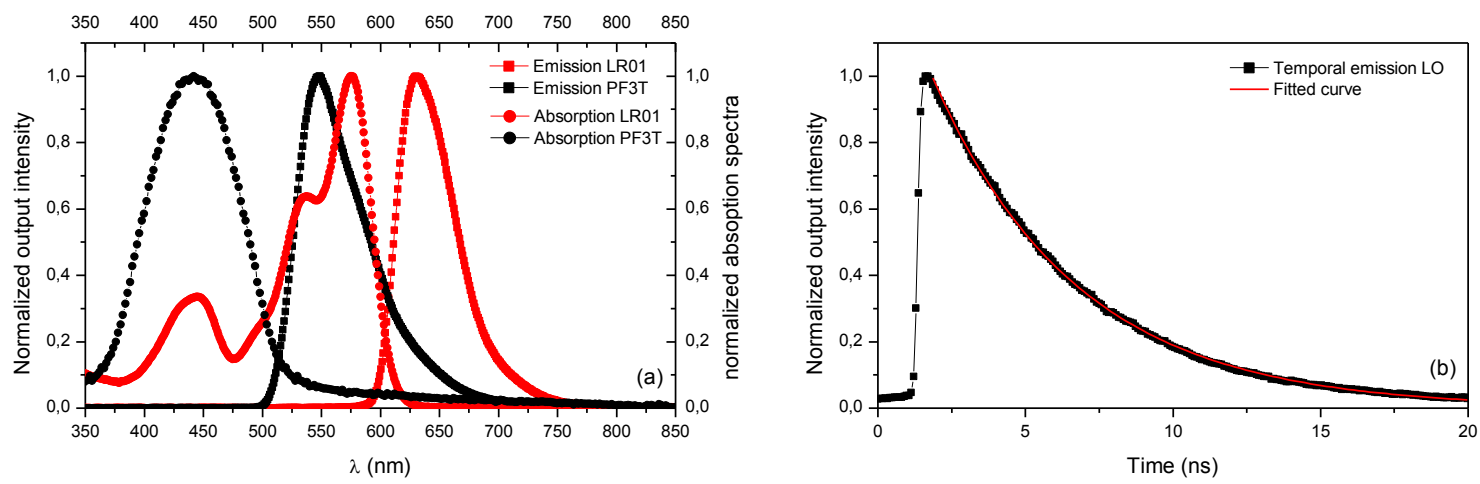

Figure 2. (a) Absorption and emission spectra of LR01 and PF3T. (b) Temporal fluorescence decay of the LO fiber for a $\lambda_{\mathrm{ex}}=457 \mathrm{~nm}$ and $\mathrm{z}_{\mathrm{ne}}=4 \mathrm{~cm}$.

Figure 3 represents the evolution of the average emission wavelength, $\lambda_{\text {avg }}$, as a function of the propagation distance. As can be observed, all fibers undergo a red-shift on their emission sprectra while the non-excited length of the fiber is increased. Such shift towards higher wavelengths is directly related to the overlap between the absorption and the emission spectra, which, as said before, is a common feature in organic dopants. Because of this overlap, successive reabsorption events can occur, and consequently, the probability that a photon may be lost by non-radiative emission increases. Therefore, for FFSC applications where the number of emitted photons plays an important role on the final efficiency, the red-shift of the emission spectrum should be as small as possible. For our fibers, Lumogen Red doped samples exhibit the smallest red-shift. However, it is seen that when the concentration of the dopant is increased, as it is the case of LR03 and LR04, the slope of the $\lambda_{\text {avg }}$ is slightly higher. The values of the average emission wavelength are gathered in Table 2.
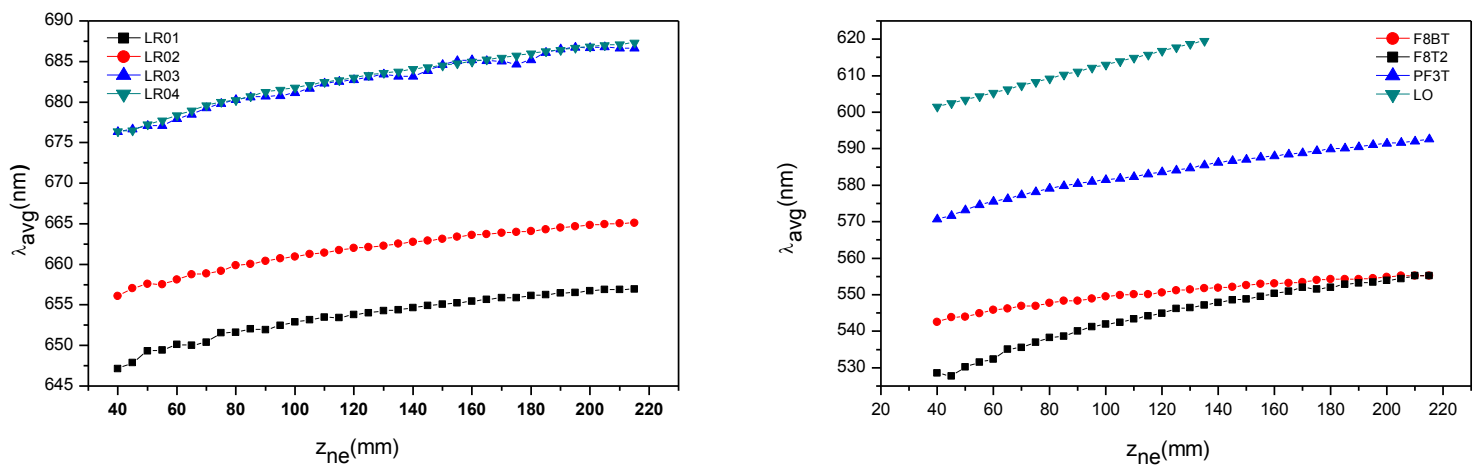

Figure 3. Evolution of the average emission wavelength as a function of the non-excited length of the fiber. $\lambda_{\mathrm{ex}}=520 \mathrm{~nm}$ for LO and LR01-04; $\lambda_{\mathrm{ex}}=457 \mathrm{~nm}$ for the conjugated polymers. $\mathrm{z}_{\mathrm{e}}=1.2 \mathrm{~mm}$.

To conclude this section, the attenuation curves of the fibers have been calculated. A characterization of the optical losses is also essential for FFSC applications to avoid extra losses when the light is propagated through the fiber. As 
mentioned before, the optical losses have been determined employing the side illumination technique. The optical loss coefficients have been calculated by measuring the decrease of the irradiance of the fluorescence spectra as the light propagation distance $\left(\mathrm{z}_{\mathrm{ne}}\right)$ is increased with a constant excitation length of $1.2 \mathrm{~mm}$. The output light measured at the fiber end for each of the desired wavelengths decays exponentially. The results obtained by fitting the experimental curves to an exponential decay are shown in Figure 4. As can be observed in this figure, the fibers doped with conjugated polymers show slightly higher values of attenuation in the region where the dopant absorption tends to be neglectable in comparison with the Lumogen Red doped ones, the F8T2 sample is the one with the highest values of attenuation.
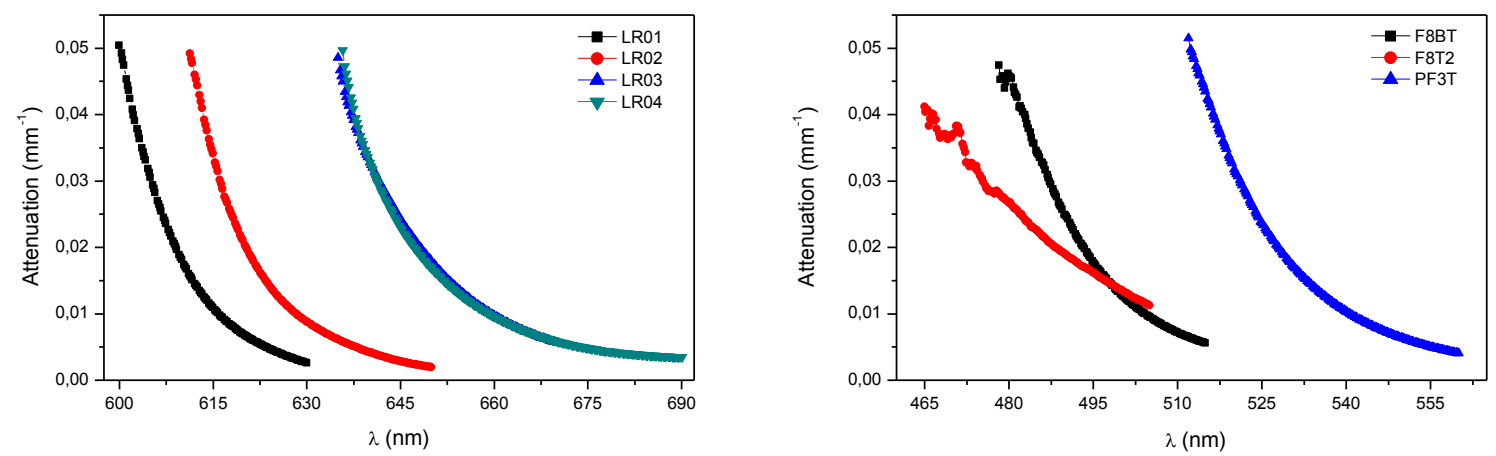

Figure 4. Linear attenuation coefficients for Lumogen Red and conjugated polymers doped fibers. $\lambda_{\mathrm{ex}}=520 \mathrm{~nm}$ for LR01-04; $\lambda_{\mathrm{ex}}=457 \mathrm{~nm}$ for the conjugated polymers.

Table 2. Stokes shift, average emission wavelength and life-time values of each of the samples.

\begin{tabular}{|c|c|c|c|c|c|c|c|c|}
\hline Fiber & LO & LR01 & LR02 & LR03 & LR04 & F8BT & F8T2 & PF3T \\
\hline $\begin{array}{c}\text { Stokes } \\
\text { Shift (nm) }\end{array}$ & 63 & 56 & 67 & 86 & 86 & 104 & 95 & 107 \\
\hline $\begin{array}{c}\text { Life-time } \\
(\mathrm{ns})\end{array}$ & $4.6 \pm 0.2$ & $7.8 \pm 0.3$ & $9.6 \pm 0.5$ & $12.1 \pm 0.4$ & $12.3 \pm 0.9$ & $3.2 \pm 0.2$ & $\begin{array}{c}0.56 \pm \\
0.05\end{array}$ & $\begin{array}{c}0.61 \pm \\
0.04\end{array}$ \\
\hline $\begin{array}{c}\lambda_{\text {avg }} \text { slope } \\
(\mathrm{nm} / \mathrm{cm})\end{array}$ & $1.9 \pm 0.02$ & $0.5 \pm 0.02$ & $0.5 \pm 0.01$ & $0.6 \pm 0.02$ & $0.6 \pm 0.02$ & $0.7 \pm 0.02$ & $1.5 \pm 0.06$ & $1.2 \pm 0.03$ \\
\hline
\end{tabular}

\subsection{Side illumination coupling efficiency}

An important feature of a FFSC relies on how much light is absorbed sideway. We have calculated the power absorbed by each of the fibers, $\mathrm{P}_{\mathrm{abs}}$, as a function of the side excitation power, $\mathrm{P}_{\text {pump. }}$. The excitation length is $1.2 \mathrm{~mm}$, which corresponds to the output beam of the femtosecond laser, and the pump power employed goes from 1 to $30 \mathrm{~mW}$. The absorbed power is calculated as the subtraction between the pump power and the power measured with a silicon photodetector just in the other side after passing through the fiber thickness of $1 \mathrm{~mm}$. We define the side illumination coupling efficiency as:

$$
\eta_{\text {SIC }}(\lambda)=\frac{p_{a b s}(\lambda)}{P_{\text {pump }}(\lambda)} \times 100(\%)
$$

The results obtained for all the fibers are gathered in Table 3. Samples have been excited at $520 \mathrm{~nm}$ and $435 \mathrm{~nm}$, which corresponds to wavelengths around their absorption peaks. The fibers with the higher $\eta_{\text {SIC }}$ turned out to be LR03 and LR04. LR02 and LO also demonstrate high enough values of $\eta_{\text {sIC }}$, but fibers doped with conjugated polymers show values below $60 \%$ in all cases. It has also been proved that there exists a strong dependence on the dopant concentration. If we focus on Lumogen Red doped fibers LR01, LR02 and LR03 with $0.0001 \mathrm{~mol} \%, 0.0005 \mathrm{~mol} \%$ and $0.03 \mathrm{~mol} \%$ 
respectively, it is observed that bigger dopant concentrations yield higher side absorption efficiencies. It could be thought that bigger dopant concentrations would always result in higher absorption efficiencies, and therefore, become more suitable for FFSC applications. However, there also exists a saturation concentration level induced by the aggregates molecules formed when high concentrations are used, which could become counterproductive [16]. Nevertheless, as the efficiencies increase with concentration, this indicates that we have not yet reached the saturation limit. Finding the most optimum dye concentration should be an important work to carry out in future research.

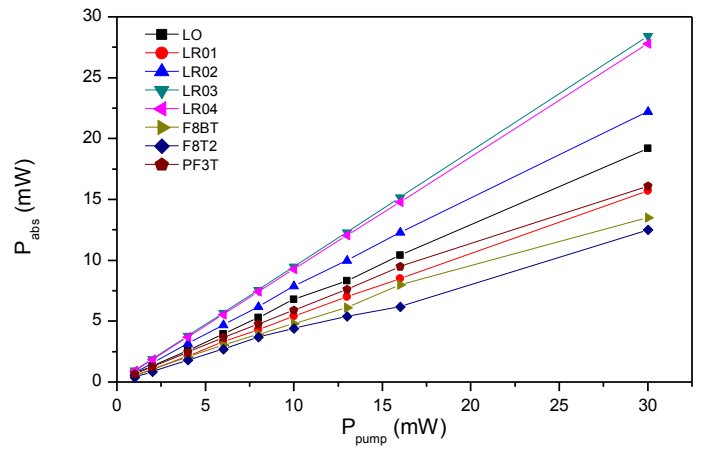

Figure 5. Power absorbed by each of the samples as a function of the excitation power at their maximum absorption wavelength. $\lambda_{\mathrm{ex}}=520 \mathrm{~nm}$ for LO and LR01-04; $\lambda_{\mathrm{ex}}=457 \mathrm{~nm}$ for the conjugated polymers.

Table 3. Values of the side illumination coupling efficiencies.

\begin{tabular}{|c|c|c|c|c|c|c|c|c|}
\hline Fiber & LO & LR01 & LR02 & LR03 & LR04 & F8BT & F8T2 & PF3T \\
\hline$\eta_{\text {SIC }}(\%)$ & 65 & 53 & 77 & 95 & 93 & 48 & 39 & 58 \\
\hline
\end{tabular}

\subsection{Fluorescence fiber solar concentrator efficiency}

Finally, we have calculated the fluorescence fiber solar concentration efficiency. We define this parameter as follows:

$$
\eta_{F F S C}=\frac{P_{\text {out }}}{P_{\text {solar }}} \times 100(\%)
$$

where $\mathrm{P}_{\text {out }}$ corresponds to the power measured at one end of the fiber and $\mathrm{P}_{\text {solar }}$ corresponds to the power emitted by the lamp on the side surface of the fiber in a wavelength range from 400 to $1100 \mathrm{~nm}$. $\mathrm{P}_{\text {solar }}$ at a distance of $36 \mathrm{~cm}$ to the fiber has been measured as $0.38 \mathrm{~kW} / \mathrm{m}^{2}$, which corresponds to approximately half a sun radiation in the range between 400 and $1100 \mathrm{~nm}$. The measurements were acquired for a total illuminated length of $4.5 \mathrm{~cm}$ and a non-excited length of $3.3 \mathrm{~cm}$. The $\eta_{\text {FFSC }}$ values for each of the fibers can be seen in Table 4 . The fiber with the higher $\eta_{\text {FFSC }}$ results to be LR03 with a value of $0.26 \%$. The fibers with the lowest light concentration capability are those doped with conjugated polymers and Lumogen Orange doped fiber. This may be caused because the low dopant concentration. It should be noted that there is a considerable difference between LR03 and LR04, which are fibers with the same dopant concentration with the only difference that LR03 has a thin cladding and LR04 is an only core fiber. In the previous section we demonstrated that these two fibers coupled light with nearly the same efficiency, as it was expected for the same dopant concentration. However, LR03 presents 2.6 times greater light concentration efficiency than LR04. From these results we can reach to the conclusion that the presence of a thin cladding is essential to minimize attenuation losses as we showed in [17]. 
These $\eta_{\text {FFSC }}$ could easily be increased by considering a multifiber system after demonstrating that the lamp emission is homogeneous for at least an area of $10 \mathrm{~cm}^{2}$. This configuration consists of 50 pieces of fibers embedded in a $10 \mathrm{~cm}$ wide and $4.5 \mathrm{~cm}$ long illuminated area. The space between each two fibers has been set as $1 \mathrm{~mm}$ in order to avoid possible shadow-caused losses between pieces. Considering that each of the pieces emit as a measured single fiber, the $\eta_{\text {FFSC }}$ would increase 50 times, yielding values of $13 \%$ in the best case.

Table 4. Experimental values of the fluorescent fiber solar concentration efficiency for a single fiber of ze $=4.5 \mathrm{~cm}$, and theoretical estimation of the $\eta_{\mathrm{FFSC}}$ of a multifiber configuration of 50 pieces.

\begin{tabular}{|c|c|c|c|c|c|c|c|c|}
\hline Fiber & LO & LR01 & LR02 & LR03 & LR04 & F8BT & F8T2 & PF3T \\
\hline$\eta_{\text {FFSC }}(\%)$ & 0.006 & 0.045 & 0.12 & 0.26 & 0.1 & 0.007 & 0.003 & 0.005 \\
\hline $\begin{array}{c}\text { Multifiber } \\
\eta_{\text {FFSC }}(\%)\end{array}$ & 0.3 & 2.25 & 6 & 13 & 5 & 0.35 & 0.15 & 0.25 \\
\hline
\end{tabular}

\section{CONCLUSIONS}

In this work, we have carried out an optical characterization of five organic dye-doped and three conjugated polymerdoped POFs focusing on future fluorescent fiber solar concentrator applications. We have seen that all fibers undergo red-shifts on their emission spectra when the non-excited length of the fiber is increased, and their emission life-times, which follow single exponential decays, are neither wavelength nor propagation distance dependent. Moreover, we have calculated the side illumination coupling efficiency, obtaining the best values for Lumogen Red doped fibers. We have observed a strong dependence with dopant concentration, yielding higher side illumination coupling efficiencies as the concentration is increased. The fluorescent fiber solar concentrator efficiency has also been experimentally measured employing a solar simulator lamp. The highest values have been achieved for the LR03 sample $(0.03$ mol\% Lumogen Red cladded fiber). It has been demonstrated that the presence of a thin cladding is essential in order to minimize the attenuation losses, and consequently, to increase the overall efficiency of the device. We have made a theoretical estimation of the $\eta_{\mathrm{FFSC}}$ for a multifiber system, which appears to provide a tangible advantage to increase the efficiency of the FFSC. From these preliminary results, it can be concluded that Lumogen Red fibers with high dopant concentration and thin cladding stacked on a multifiber system seem to be a good candidate for possible applications in the field of luminescent solar concentrators.

\section{REFERENCES}

[1] Weber, W. H., Lambe, J., "Luminescent greenhouse collector for solar radiation,” Appl. Opt. 15(10), 2299, Optical Society of America (1976).

[2] Colantuono, G., Buckley, A., Erdelyi, R., "Ray-Optics Modelling of Rectangular and Cylindrical 2-Layer Solar Concentrators," J. Light. Technol. 31(7), 1033-1044 (2013).

[3] McIntosh, K. R., Yamada, N., Richards, B. S., "Theoretical comparison of cylindrical and square-planar luminescent solar concentrators," Appl. Phys. B 88(2), 285-290, Springer-Verlag (2007).

[4] Wang, T., Yu, B., Chen, B., Hu, Z., Luo, Y., Zou, G., Zhang, Q., “A theoretical model of a cylindrical luminescent solar concentrator with a dye-doping coating," J. Opt. 15(5), 55709, IOP Publishing (2013).

[5] Correia, S. F. H., Lima, P. P., André, P. S., Ferreira, M. R. S., Carlos, L. A. D., "High-efficiency luminescent solar concentrators for flexible waveguiding photovoltaics," Sol. Energy Mater. Sol. Cells 138, 51-57 (2015).

[6] Inman, R. H., Shcherbatyuk, G. V., Medvedko, D., Gopinathan, A., Ghosh, S., "Cylindrical luminescent solar concentrators with near-infrared quantum dots," Opt. Express 19(24), 24308, Optical Society of America (2011).

[7] Purcell-Milton, F., Gun'ko, Y. K., "Quantum dots for Luminescent Solar Concentrators," J. Mater. Chem. 22(33), 16687 (2012).

[8] Wilson, L. R., Rowan, B. C., Robertson, N., Moudam, O., Jones, A. C., Richards, B. S., "Characterization and 
reduction of reabsorption losses in luminescent solar concentrators.," Appl. Opt. 49(9), 1651-1661 (2010). Sholin, V., Olson, J. D., Carter, S. A., "Semiconducting polymers and quantum dots in luminescent solar concentrators for solar energy harvesting," J. Appl. Phys. 101(12) (2007).

[10] Nolasco, M. M., Vaz, P. M., Freitas, V. T., Lima, P. P., André, P. S., Ferreira, R. a S., Vaz, P. D., Ribeiro-Claro, P., Carlos, L. D., "Engineering highly efficient Eu(iii)-based tri-ureasil hybrids toward luminescent solar concentrators," J. Mater. Chem. A 1(25), 7339-7350 (2013).

[11] Wang, T., Zhang, J., Ma, W., Luo, Y., Wang, L., Hu, Z., Wu, W., Wang, X., Zou, G., et al., "Luminescent solar concentrator employing rare earth complex with zero self-absorption loss," Sol. Energy 85(11), 2571-2579 (2011).

[12] Wu, W., Wang, T., Wang, X., Wu, S., Luo, Y., Tian, X., Zhang, Q., "Hybrid solar concentrator with zero selfabsorption loss," Sol. Energy 84(12), 2140-2145 (2010).

[13] Rowan, B. C., Wilson, L. R., Richards, B. S., "Advanced material concepts for luminescent solar concentrators," IEEE J. Sel. Top. Quantum Electron. 14(5), 1312-1322 (2008).

[14] Earp, A. A., Smith, G. B., Franklin, J., Swift, P., "Optimisation of a three-colour luminescent solar concentrator daylighting system," Sol. Energy Mater. Sol. Cells 84(1), 411-426 (2004).

[15] Bazzana L., Lanzani G., Xia R., Morgado J., S. S. and L. D. G., "Plastic optical fibers with embedded organic semiconductors for signal amplification," Proc. 16th Int. Plast. Opt. Fibers Conf., Torino, 327 (2001).

[16] Costela, A., García-Moreno, I., Sastre, R., "Polymeric solid-state dye lasers: Recent developments," Phys. Chem. Chem. Phys. 5(21), 4745-4763 (2003).

[17] Parola, I., Illarramendi, M. A., Ayesta, I., Arrue, J., Jiménez, F., Zubia, J., Diamant, S., Palchik, O., “Analysis of cladded and uncladded dye-doped step-index polymer optical fibers," Organic Photonics VII proceecings of SPIE, 989504 (2016).

\section{ACKNOWLEDGMENTS}

This work has been funded in part by the Fondo Europeo de Desarrollo Regional (FEDER); by the Ministerio de Economía y Competitividad under project TEC2015-638263-C03-1-R; by the Gobierno Vasco/Eusko Jaurlaritza under projects IT933-16 and ELKARTEK (KK-2016/0030 and KK-2016/0059); by the University of the Basque Country UPV/EHU under program UFI11/16; and by Ministerio de Economía y Competitividad under project MAT2014-57429R. The work carried out by I. Parola has been funded by a research grant given by the Departamento de Educación, Política Lingüística y Cultura del Gobierno Vasco/Eusko Jaurlaritza for her PhD thesis. 